\title{
Quadratic performance of generalized first-order systems
}

\author{
Robert van der Geest \\ University of Twente \\ Faculty of Applied Mathematics \\ P.O.Box 217, 7500 AE Enschede, Netherlands \\ Email: vdgeest@math.utwente.nl
}

\author{
Anders Rantzer \\ Lund Institute of Technology \\ Department of Automatic Control \\ P.O.Box 118, S 22100 Lund, Sweden \\ Email: rantzer@control.lth.se
}

\begin{abstract}
In this note we formulate the Kalman-Yakubovič-Popov Lemma for generalized first-order systems, both in continuous- and discrete-time.
\end{abstract}

\section{Introduction}

The Kalman-Yakubovič-Popov (KYP) Lemma is a primary tool for the analysis of linear systems in statespace description. It provides a link between quadratic performance questions and the existence of a solution to a Linear Matrix Inequality (LMI). A demonstration of this connection, and some background about the KYP Lemma may be found in Willems [4].

In this note we formulate the KYP Lemma for continuous-time, generalized first-order systems of the form

$$
G \dot{w}=F w
$$

where $w \in \mathcal{L}_{2}\left(\mathbb{R}, \mathbb{R}^{q}\right)$ are the variables associated with the system, and $G$ and $F$ are real-valued, $p$ by $q$ matrices. Such a description allows for specification of a number of algebraic constraints, i.e., constraints of the type

$$
H w=0 \text {, }
$$

where $H$ is a real-valued matrix. In this respect (1) is a generalization of a state-space description, which consists of dynamic restrictions only. Note also that contrary to what happens in state-space theory, we do not a priori split up the variables $w$ into inputs and outputs. An introduction to different kinds of firstorder models, and some motivation for studying them may be found in Kuijper [1].

The quadratic performance criterion that we are interested in has the form

$$
\int_{-\infty}^{\infty} w^{T}(t) M w(t) d t \leq 0
$$

where $M$ is a symmetric, real-valued, $q$ by $q$ matrix. It is instructive to think of the integral in (3) as the energy enclosed in the signal $w$. It turns out that a controllable system (1), without algebraic constraints, satisfies (3) if and only if there exists a symmetric solution $P$ to the LMI

$$
M+F^{T} P G+G^{T} P F \leq 0 .
$$
tem

Analogously, it turns out that the discrete-time sys-

$$
G w(t+1)=F w(t)
$$

satisfies the performance criterion

$$
\sum_{-\infty}^{\infty} w^{T}(t) M w(t) \leq 0
$$

if and only if there exists a symmetric solution $P$ to the discrete-time LMI

$$
M+F^{T} P F-G^{T} P G \leq 0 .
$$

When the system description (1) or (5) includes algebraic constraints, the behaviour of the system is restricted to a linear subspace, and we show how the quadratic performance problem may be reduced to an equivalent problem on a subspace.

\section{Quadratic performance}

Before we formulate the main results, we first characterize controllability of a system in kernel representation (Willems [5]).

Lemma 2.1 The system $R\left(\frac{d}{d t}\right) w=0$ is controllable if and only if the rank of $R(\lambda)$ is constant for all $\lambda \in \mathbb{C}$.

The KYP Lemma for continuous-time, generalized first-order systems is formulated as follows.

Theorem 2.2 Assume that the system $G \dot{w}=F w$ is controllable, and that the matrix $G$ has full row-rank. Then the following two statements are equivalent:

- For all $w \in \mathcal{L}_{2}\left(\mathbb{R}, \mathbb{R}^{q}\right)$ such that $G \dot{w}=F w$,

$$
\int_{-\infty}^{\infty} w^{T}(t) M w(t) d t \leq 0 .
$$

- There exists a symmetric solution $P$ to the $L M I$

$$
M+F^{T} P G+G^{T} P F \leq 0 .
$$

Proof: It is possible to prove the result directly along the lines of the proof in Rantzer [2]. Here we convert the problem into state-space form instead. By Parseval's Theorem, (8) is equivalent to

$$
\begin{aligned}
\forall w \in \mathcal{L}_{2}\left(\mathbb{R}, \mathbb{R}^{q}\right) & \text { s.t. } G \dot{w}=F w: \\
& \int_{-\infty}^{\infty} \hat{w}^{T}(-i \omega) M \hat{w}(i \omega) d \omega \leq 0
\end{aligned}
$$


By a continuity argument, (10) is equivalent to

$$
\begin{aligned}
\forall \omega \in \mathbb{R}: \forall v \in \mathbb{C}^{q} \text { s.t. }(i \omega G-F) v=0: \\
\qquad v^{*} M v \leq 0 .
\end{aligned}
$$

Since $G$ has full row-rank, there exist invertible matrices $U$ and $V$ such that $U G V=\left(\begin{array}{ll}I & 0\end{array}\right)$. Define

$$
U F V=:\left(\begin{array}{ll}
A & B
\end{array}\right), \text { and } V^{-1} v=:\left(\begin{array}{l}
x \\
u
\end{array}\right) .
$$

Then $(A, B)$ is a controllable pair, and (11) is equivalent to

$$
\begin{array}{r}
\forall \omega \in \mathbb{R}: \forall\left(\begin{array}{l}
x \\
u
\end{array}\right) \in \mathbb{C}^{q} \text { s.t. } i \omega x=A x+B u: \\
\qquad\left(\begin{array}{l}
x \\
u
\end{array}\right)^{*} V^{T} M V\left(\begin{array}{l}
x \\
u
\end{array}\right) \leq 0 .
\end{array}
$$

The KYP Lemma for continuous-time systems in statespace form may be found in Yakubovich [7]. By this Lemma, (13) is equivalent to

$$
\begin{aligned}
& \exists Q=Q^{T} \text { s.t. } \\
& \qquad V^{T} M V+\left(\begin{array}{cc}
A^{T} Q+Q A & Q B \\
B^{T} Q & 0
\end{array}\right) \leq 0 .
\end{aligned}
$$

Take $P=U^{T} Q U$. Then (14) is equivalent to (9).

Remark: Note the similarity between Theorem 2.2 and the so-called Projection Lemma, see e.g. Scherer [3].

The discrete-time counterpart of Theorem 2.2 is the following.

Theorem 2.3 Assume that the system $G w(t+1)=$ $F w(t)$ is controllable, and that the matrix $G$ has full row-rank. Then the following two statements are equivalent:

- For all $w \in \ell_{2}\left(\mathbb{R}, \mathbb{R}^{q}\right)$ such that $G w(t+1)=$ $F w(t)$,

$$
\sum_{-\infty}^{\infty} w^{T}(t) M w(t) \leq 0 .
$$

- There exists a symmetric solution P to the LMI

$$
M+F^{T} P F-G^{T} P G \leq 0 .
$$

Proof: The proof is analogous to that of Theorem 2.2, using the KYP Lemma for discrete-time systems in state-space form.

\section{Algebraic constraints}

The condition that $G$ has full row-rank is equivalent to excluding algebraic constraints on the system (1). If the system description does include algebraic constraints, the behaviour of (1) is restricted to live on a linear subspace of $\mathbb{R}^{\mathrm{q}}$, and Theorem 2.2 should be adjusted accordingly.
Theorem 3.1 Say that the description $G \dot{w}=F w$ includes algebraic constraints restricting the behaviour to image $(W) \subseteq \mathbb{R}^{\mathrm{q}}$. Assume that the system is controllable on image $(W)$. Then the following two statements are equivalent:

- For all $w \in \mathcal{L}_{2}\left(\mathbb{R}, \mathbb{R}^{q}\right)$ such that $G \dot{w}=F w$,

$$
\int_{-\infty}^{\infty} w^{T}(t) M w(t) d t \leq 0
$$

- There exists a symmetric solution P to the LMI

$$
W^{T}\left(M+F^{T} P G+G^{T} P F\right) W \leq 0 .
$$

\subsection{Reduction procedure}

The following procedure may be used to detect any algebraic constraints in the description $G \dot{w}=F w$. Assume that the matrix $G$ does not have full row-rank. Then there exists an invertible matrix $U$ such that

$$
U G=\left(\begin{array}{c}
\tilde{G} \\
0
\end{array}\right), \text { and } U F=\left(\begin{array}{c}
\tilde{F} \\
H
\end{array}\right)
$$

where $\tilde{G}$ has full row-rank. The behaviour of the system is equivalently described as

$$
\tilde{G} \dot{w}=\tilde{F} w \text {, and } H w=0 .
$$

The algebraic constraints $H w=0$ may be rewritten in image representation as $w=$ image $\left(H^{\perp}\right)^{T}$.

Remark: In Theorem 2.2, the quadratic function

$$
f(w):=w^{T} G^{T} P G w
$$

is a storage function in the sense of dissipative systems theory. It is possible to prove the equivalence in Theorem 2.2 by finding this storage function using the results in a forthcoming paper by Willems and Trentelman $[6]$.

\section{References}

[1] M. Kuijper, First-order representations of linear systems, Birkhäuser, 1994.

[2] A. Rantzer, 'On the Kalman-Yakubovich-Popov lemma,' Systems 8 Control Letters, Vol.28, 1996, pp.710.

[3] C.W. Scherer, 'Mixed $H_{2} / H_{\infty}$ control,' in: A. Isidori (ed.), Trends in control: a European perspective, Springer-Verlag, 1995.

[4] J.C. Willems, 'Least squares stationary optimal control and the algebraic Riccati equation,' IEEE T$A C$, Vol.16, No.6, Dec.1971, pp.621-634.

[5] J.C. Willems, 'Paradigms and puzzles in the theory of dynamical systems,' IEEE T-AC, Vol.36, 1991, pp.259-294.

[6] J.C. Willems and H.L. Trentelman, 'On quadratic differential forms,' preprint.

[7] V.A. Yakubovich, 'A frequency theorem in control theory,' translated from Sibirskii Matematicheskii Zhurnal, Vol.14, No.2, 1973, pp.384-419. 